\title{
Nonstationary Gabor Frames - Approximately Dual Frames and Reconstruction Errors
}

\author{
Monika Dörfler, Ewa Matusiak *†
}

October 31, 2018

\begin{abstract}
Nonstationary Gabor frames, recently introduced in adaptive signal analysis, represent a natural generalization of classical Gabor frames by allowing for adaptivity of windows and lattice in either time or frequency. Due to the lack of a complete lattice structure, perfect reconstruction is in general not feasible from coefficients obtained from nonstationary Gabor frames. In this paper it is shown that for nonstationary Gabor frames that are related to some known frames for which dual frames can be computed, good approximate reconstruction can be achieved by resorting to approximately dual frames. In particular, we give constructive examples for so-called almost painless nonstationary frames, that is, frames that are closely related to nonstationary frames with compactly supported windows. The theoretical results are illustrated by concrete computational and numerical examples.
\end{abstract}

Keywords: adaptive representations, nonorthogonal expansions, irregular Gabor frames, reconstruction, approximately dual frame

\section{Introduction}

Adapted and adaptive signal representation have received increasing interest over the past few years. As opposed to classical approaches such as the short-time Fourier

${ }^{*}$ This work was supported by the WWTF project Audiominer (MA09-24)

${ }^{\dagger}$ The authors are with the Department of Mathematics, NuHAG, University of Vienna, Nordbergstrasse 15, 1090 Wien, Austria (e-mail: monika.doerfler@univie.ac.at, ewa.matusiak@univie.ac.at) 
transform (STFT) or wavelet transform, adaptive representations allow for a variation of parameters such as window width or sampling density over time, frequency or both. Changing parameters in the frequency domain leads, for example, to non-uniform filter banks while adapting window width and sampling density in time is reminiscent of the approach suggested in the construction of nonuniform lapped transforms. Transforms featuring simultaneous adaptivity in time and frequency are notoriously difficult to construct and implement, cp. [15, 7, 12]; however they have shown to be useful in some applications, cf. [14. On the other hand, fast and efficient implementations exist for representations with adaptivity in only time or frequency. One recent method to obtain this kind of representations is represented by nonstationary Gabor frames, first suggested in [11] and further developed in [1, 18, 10]. All the known implementations rely on compactness of the used analysis window in either time of frequency. This assumption allows for usage of tools developed for painless non-orthogonal expansions [6]. While a priori very convenient, the restriction to using compactly supported windows in the domain for which one wishes a flexible representation can be undesirable. As an example, we mention the construction of nonuniform filter banks via nonstationary Gabor frames, in which case this restriction forbids finite impulse response (FIR) filters; the latter are, however, imperative for real-time processing applications.

In the current contribution, we therefore go beyond the results presented in the references above and consider nonstationary Gabor frames with fast decay but unbounded support. The existence of this kind of frames was shown in [8]. Here we are concerned with methods for approximate reconstruction for these adaptive systems.

\section{Notation and Preliminaries}

Given a non-zero function $g \in L^{2}(\mathbb{R})$, a modulation, or frequency shift, operator $M_{b l}$ is defined by $M_{b l} g(t):=e^{2 \pi i b l t} g(t)$, and time shift operator $T_{a k}$ by $T_{a k} g(t):=g(t-a k)$. A composition, $g_{k, l}=M_{b l} T_{a k} g(t):=e^{2 \pi i b l t} g(t-a k)$ is a time-frequency shift operator.

The set $\mathcal{G}(g, a, b)=\left\{g_{k, l}: k, l \in \mathbb{Z}\right\}$ is called a Gabor system for any real, positive $a, b . \mathcal{G}(g, a, b)$ is a Gabor frame for $L^{2}(\mathbb{R})$, if there exist frame bounds $0<A \leq B<\infty$ such that for every $f \in L^{2}(\mathbb{R})$ we have

$$
A\|f\|_{2}^{2} \leq \sum_{k, l \in \mathbb{Z}}\left|\left\langle f, g_{k, l}\right\rangle\right|^{2} \leq B\|f\|_{2}^{2} .
$$


When working with irregular grids, we assume that the sampling points form a separated set: a set of sampling points $\left\{a_{k}: k \in \mathbb{Z}\right\}$ is called $\delta$-separated, if $\left|a_{k}-a_{m}\right|>\delta$ for $a_{k}, a_{m}$, whenever $k \neq m$. $\chi_{I}$ will denote the characteristic function of the interval $I$.

A convenient class of window functions for time-frequency analysis on $L^{2}(\mathbb{R})$ is the Wiener space.

Definition 1. A function $g \in L^{\infty}(\mathbb{R})$ belongs to the Wiener space $W\left(L^{\infty}, \ell^{1}\right)$ if

$$
\|g\|_{W\left(L^{\infty}, \ell^{1}\right)}:=\sum_{k \in \mathbb{Z}} \operatorname{ess} \sup _{t \in Q}|g(t+k)|<\infty, \quad Q=[0,1]
$$

For $g \in W\left(L^{\infty}, \ell^{1}\right)$ and $\delta>0$ we have [9]

$$
\operatorname{ess}_{\sup } \sum_{t \in \mathbb{R}} \sum_{k \in \mathbb{Z}}|g(t-\delta k)| \leq\left(1+\delta^{-1}\right)\|g\|_{W\left(L^{\infty}, \ell^{1}\right)}
$$

For $f \in L^{2}(\mathbb{R})$ we use the following normalization of the Fourier transform, which we denote by $\mathcal{F}$ :

$$
\mathcal{F} f(\omega)=\widehat{f}(\omega)=\int_{\mathbb{R}} f(t) e^{-2 \pi i \omega t} d t
$$

\section{$3 \quad$ Nonstationary Gabor Frames}

Nonstationary Gabor systems are a generalization of classical Gabor systems of regular time-frequency shifts of a single window function.

Definition 2. Let $\mathbf{g}=\left\{g_{k} \in W\left(L^{\infty}, \ell^{1}\right): k \in \mathbb{Z}\right\}$ be a set of window functions and let $\mathbf{b}=\left\{b_{k}: k \in \mathbb{Z}\right\}$ be a corresponding sequence of frequency-shift parameters. Set $g_{k, l}=M_{b_{k} l} g_{k}$. Then, the set

$$
\mathcal{G}(\mathbf{g}, \mathbf{b})=\left\{g_{k, l}: k, l \in \mathbb{Z}\right\}
$$

is called a nonstationary Gabor (NSG) system.

In generalization of regular Gabor frames, for which $g_{k}=T_{a k} g$, we will usually assume that the windows $g_{k}$ are localized around points $a_{k}$ in a separated set of timesampling points $\left\{a_{k}: k \in \mathbb{Z}\right\}$. Further, we will always make the assumption that the frequency sampling parameters $b_{k}$ are positive numbers contained in a closed interval, i.e. $b_{k} \in\left[b_{L}, b_{U}\right] \subset \mathbb{R}^{+}$for all $k \in \mathbb{Z}$. 
To every collection (3) we associate the analysis operator $C_{g}$ given by $\left(C_{g} f\right)_{k, l}=$ $\left\langle f, g_{k, l}\right\rangle$, and synthesis operator $U_{g}$, where $U_{g} c=\sum_{k, l \in \mathbb{Z}} c_{k, l} g_{k, l}$ and $c \in \ell^{2}$. For two Gabor systems $\mathcal{G}(\mathbf{g}, \mathbf{b})$ and $\mathcal{G}(\gamma, \mathbf{b})$ the composition $S_{g, \gamma}=U_{\gamma} C_{g}$,

$$
S_{g, \gamma} f=\sum_{k, l \in \mathbb{Z}}\left\langle f, M_{l b_{k}} g_{k}\right\rangle M_{l b_{k}} \gamma_{k}
$$

admits a Walnut representation for all $f \in L^{2}(\mathbb{R}),[]$ :

$$
S_{g, \gamma} f(t)=\sum_{k, l \in \mathbb{Z}} b_{k}^{-1} \overline{g_{k}\left(t-l b_{k}^{-1}\right)} \gamma_{k}(t) f\left(t-l b_{k}^{-1}\right) .
$$

We will frequently use the following correlation functions of a pair of Gabor systems:

$$
G_{l}^{g, \gamma}(t)=\sum_{k \in \mathbb{Z}} b_{k}^{-1}\left|g_{k}\left(t-l b_{k}^{-1}\right)\right|\left|\gamma_{k}(t)\right|, \text { for } l \in \mathbb{Z} .
$$

Note that this definition is asymmetric with respect to $\mathrm{g}$ and $\boldsymbol{\gamma}$. Using this notation, we obtain the following bounds for the frame operator (4):

$$
\left\|S_{g, \gamma}\right\|^{2} \leq \operatorname{ess} \sup \sum_{l \in \mathbb{Z}} G_{l}^{g, \gamma} \cdot \operatorname{ess} \sup \sum_{l \in \mathbb{Z}} G_{l}^{\gamma, g} .
$$

By inspection of (5), we note that the summands corresponding to $l \neq 0$ may be seen as the off-diagonal entries of the frame operator. We thus isolate the diagonal part

$$
G_{0}^{g, \gamma}(t)=\sum_{k \in \mathbb{Z}} b_{k}^{-1}\left|g_{k}(t)\right|\left|\gamma_{k}(t)\right|
$$

and denote the off-diagonal entries as follows:

$$
R_{g, \gamma}=\operatorname{ess} \sup \sum_{l \in \mathbb{Z} \backslash\{0\}} \sum_{k \in \mathbb{Z}} b_{k}^{-1}\left|g_{k}\left(\cdot-l b_{k}^{-1}\right)\right|\left|\gamma_{k}(\cdot)\right| .
$$

Note that, if $\mathbf{g}=\boldsymbol{\gamma}$, then the diagonal part of the frame operator $S_{g, g}$ is equal to $G_{0}^{g, g}$. Using this notation, we obtain the following additional bound:

$$
\left\langle S_{g, \gamma} f, f\right\rangle\|f\|_{2}^{-2} \leq \operatorname{ess} \sup G_{0}^{g, \gamma}+\sqrt{R_{g, \gamma} \cdot R_{\gamma, g}},
$$

Bessel sequences are of particular importance in the theory of frames and Riesz bases, [5, 19]. In the regular Gabor case, where $g_{k}(t)=g(t-a k)$ for some $a>0$, it is sufficient to assume $g \in W\left(L^{\infty}, \ell^{1}\right)$ to obtain a Bessel sequence. We next provide a generalization of this property to NSG frames. 
Proposition 3.1. Let $\mathcal{G}(\mathbf{g}, \mathbf{b})$ be a NSG system. If $g_{k} \in W\left(L^{\infty}, \ell^{1}\right)$ for all $k \in \mathbb{Z}$ with $\sup _{k \in \mathbb{Z}}\left\|g_{k}\right\|_{W\left(L^{\infty}, \ell^{1}\right)}$ bounded, and $\sum_{k \in \mathbb{Z}}\left|g_{k}(t)\right| \leq B$ almost everywhere for some $B<\infty$, then the sequence $g_{k, l}$ is a Bessel sequence.

Proof. Let $f \in L^{2}(\mathbb{R})$. Then by the assumption on the windows $g_{k}$ and estimate (7)

$$
\begin{aligned}
\sum_{k, l \in \mathbb{Z}}\left|\left\langle f, g_{k, l}\right\rangle\right|^{2} & =\left\langle S_{g, g} f, f\right\rangle \leq\|f\|_{2}^{2} \cdot \operatorname{ess} \sup \sum_{l \in \mathbb{Z}} G_{l}^{g, g} \\
& =\|f\|_{2}^{2} \cdot \operatorname{ess} \sup \sum_{k \in \mathbb{Z}} b_{k}^{-1}\left|g_{k}(\cdot)\right| \sum_{l \in \mathbb{Z}}\left|g_{k}\left(\cdot-l b_{k}^{-1}\right)\right| \\
& \leq\|f\|_{2}^{2} \cdot \operatorname{ess} \sup \sum_{k \in \mathbb{Z}} b_{k}^{-1}\left|g_{k}(\cdot)\right|\left(1+b_{k}^{-1}\right)\left\|g_{k}\right\|_{W\left(L^{\infty}, \ell^{1}\right)} \\
& \leq\|f\|_{2}^{2} \cdot B \cdot \sup _{k \in \mathbb{Z}}\left[\left(1+b_{k}\right)\left\|g_{k}\right\|_{W\left(L^{\infty}, \ell^{1}\right)}\right] .
\end{aligned}
$$

Given a frame, it is well known that there exists at least one dual frame $\mathcal{G}(\boldsymbol{\gamma}, \mathbf{b})$ such that

$$
f=\sum_{k, l \in \mathbb{Z}}\left\langle f, \gamma_{k, l}\right\rangle g_{k, l}, \quad \text { for all } f \in L^{2}(\mathbb{R}) .
$$

The canonical dual frame is given by $\gamma_{k, l}=S^{-1} g_{k, l}$. In the regular Gabor case, the dual frames are again Gabor frames, i.e., they consist of time-frequency shifted versions of one dual window. This is due to the fact, that the frame operator $S$ commutes with time-frequency shifts, hence $\gamma_{k, l}=S^{-1} g_{k, l}=S^{-1} M_{b l} T_{a k} g=M_{b l} T_{a k} S^{-1} g=$ $M_{b l} T_{a k} \gamma$. In general, we cannot expect, that the dual frame of a NSG frame is again a NSG frame.

However, even in the case of regular Gabor frames, it is often difficult to calculate a dual frame explicitly. For that reason, alternative possibilities to obtain perfect or approximate reconstruction have been proposed, [4, 2]. The following lemma quantifies the reconstruction error using general pairs of Bessel sequences.

Lemma 3.2. Let $\mathcal{G}(\mathbf{g}, \mathbf{b})$ and $\mathcal{G}(\boldsymbol{\gamma}, \mathbf{b})$ be two Bessel sequences. Then

$$
\left\|I-S_{g, \gamma}\right\| \leq\left\|1-\sum_{k \in \mathbb{Z}} b_{k}^{-1} \overline{g_{k}} \gamma_{k}\right\|_{\infty}+\sqrt{R_{g, \gamma} \cdot R_{\gamma, g}} .
$$

Proof. Starting from the Walnut representation of $S_{g, \gamma}$, we estimate using CauchySchwartz inequality for sums and integrals and, since all summands have absolute value, Fubini's theorem to justify changing the order of summation and integral: 


$$
\begin{aligned}
\left|\left\langle f-S_{g, \gamma} f, f\right\rangle\right| & =\left|\left\langle f-\sum_{k \in \mathbb{Z}} b_{k}^{-1} \overline{g_{k}} \gamma_{k} f, f\right\rangle-\left\langle\sum_{l \in \mathbb{Z} \backslash\{0\}} \sum_{k \in \mathbb{Z}} b_{k}^{-1} \gamma_{k}\left(\cdot \overline{g_{k}\left(\cdot-l b_{k}^{-1}\right.}\right) f\left(\cdot-l b_{k}^{-1}\right), f\right\rangle\right| \\
& \leq\left\|1-\sum_{k \in \mathbb{Z}} b_{k}^{-1} \overline{g_{k}} \gamma_{k}\right\|_{\infty}\|f\|_{2}^{2}+\sqrt{R_{g, \gamma} \cdot R_{\gamma, g}}\|f\|_{2}^{2}
\end{aligned}
$$

since

$$
\begin{aligned}
& \left|\left\langle\sum_{\substack{k \in \mathbb{Z} \\
l \in \mathbb{Z} \backslash\{0\}}} b_{k}^{-1} \gamma_{k}(\cdot) \overline{g_{k}\left(\cdot-l b_{k}^{-1}\right.}\right) f\left(\cdot-l b_{k}^{-1}\right), f\right\rangle \mid \\
& \leq \sum_{\substack{k \in \mathbb{Z} \\
l \in \mathbb{Z} \backslash\{0\}}} b_{k}^{-1} \int_{\mathbb{R}}\left|\gamma_{k}(t)\right|\left|g_{k}\left(t-l b_{k}^{-1}\right)\right|\left|f\left(t-l b_{k}^{-1}\right)\right||f(t)| d t \\
& \leq \sum_{\substack{k \in \mathbb{Z} \\
l \in \mathbb{Z} \backslash\{0\}}} b_{k}^{-1}\left[\int_{\mathbb{R}}\left|g_{k}\left(t-l b_{k}^{-1}\right)\right|\left|\gamma_{k}(t)\right|\left|f\left(t-l b_{k}^{-1}\right)\right|^{2} d t\right]^{1 / 2}\left[\int_{\mathbb{R}}\left|g_{k}\left(t-l b_{k}^{-1}\right)\right|\left|\gamma_{k}(t)\right||f(t)|^{2} d t\right]^{1 / 2} \\
& \leq\left[\int_{\mathbb{R}}|f(t)|^{2} \sum_{\substack{k \in \mathbb{Z} \\
l \in \mathbb{Z} \backslash\{0\}}} b_{k}^{-1}\left|g_{k}(t)\right|\left|\gamma_{k}\left(t-l b_{k}^{-1}\right)\right| d t\right]^{1 / 2}\left[\int_{\mathbb{R}}|f(t)|^{2} \sum_{\substack{k \in \mathbb{Z} \\
l \in \mathbb{Z} \backslash\{0\}}} b_{k}^{-1}\left|\gamma_{k}(t)\right|\left|g_{k}\left(t-l b_{k}^{-1}\right)\right| d t\right]^{1 / 2}
\end{aligned}
$$

A special class of NSG systems are collections of compactly supported windows. They were first addressed in [1]. The collection $\mathcal{G}(\mathbf{g}, \mathbf{b})$ with windows $g_{k}$ being compactly supported with $\left|\operatorname{supp} g_{k}\right| \leq \frac{1}{b_{k}}$ for all $k$ is a frame for $L^{2}(\mathbb{R})$ if there exist constants $A>0$ and $B<\infty$ such that

$$
A \leq G_{0}^{g, g}(t) \leq B \text { a.e. . }
$$

In this situation, $\mathcal{G}(\mathbf{g}, \mathbf{b})$ is called painless $N S G$ frame. The canonical dual atoms are given by $\gamma_{k, l}=M_{l b_{k}}\left(G_{0}^{g, g} g\right)^{-1} g_{k}$. Note again that, in general, we may have $\gamma_{k, l}=$ $S^{-1}\left(M_{l b_{k}} g_{k}\right) \neq M_{l b_{k}}\left(S^{-1} g_{k}\right)$. If $b_{k}=b$ for all $k$, then the frame operator commutes with the frequency-shifts and the dual frame is an NSG frame.

The existence of NSG frames with not necessarily compactly supported windows was established in [8]. For these frames, finding canonical dual frames requires the inversion of the frame operator. This computation is expensive since the operator has considerably less structure than the frame operator in the classical, regular Gabor frame case, for which fast algorithms now exist, [17, 13, 16]. To circumvent the problem, we suggest the use of windows other than canonical duals to obtain sufficiently good approximate reconstruction. 


\section{Approximately dual atoms}

The notion of approximately dual pairs was discussed in [4]. For NSG Bessel sequences we adapt their definition as follows.

Definition 3. Two Bessel sequences $\mathcal{G}(\mathbf{g}, \mathbf{b})$ and $\mathcal{G}(\boldsymbol{\gamma}, \mathbf{b})$ are said to be approximately dual frames if $\left\|I-S_{g, \gamma}\right\|<1$ or $\left\|I-S_{\gamma, g}\right\|<1$.

Note that the two conditions given in the definition are equivalent since

$$
\left\|I-S_{g, \gamma}\right\|=\left\|I-C_{g} U_{\gamma}\right\|=\left\|I-U_{\gamma}^{*} C_{g}^{*}\right\|=\left\|I-C_{\gamma} U_{g}\right\|=\left\|I-S_{\gamma, g}\right\| .
$$

In Definition 3 it is implicitly stated that, if two Bessel sequences are approximately dual frames, then each of them is a frame. This result was proved in [4] for general frames and it will be useful to reformulate the conditions for the existence of NSG frame, given in [8], in the context of approximately dual frames.

Proposition 4.1. Let $\mathcal{G}(\mathbf{g}, \mathbf{b})$ be a Bessel sequence with Bessel bound $B$ and $0<$ $A_{1} \leq \sum_{k \in \mathbb{Z}}\left|g_{k}(t)\right|^{2} \leq A_{2}<\infty$ a.e. for some positive constants $A_{1}, A_{2}$.

i) The multiplication operator $G_{0}^{g, g}$ is invertible a.e. and, for $\gamma_{k}=\left(G_{0}^{g, g}\right)^{-1} g_{k}$,

$$
\left\|I-S_{g, \gamma}\right\| \leq \frac{R_{g, g}}{\operatorname{essinf} G_{0}^{g, g}}
$$

ii) If

$$
R_{g, g}<\operatorname{ess} \inf G_{0}^{g, g}
$$

then $\mathcal{G}(\mathbf{g}, \mathbf{b})$ and $\mathcal{G}(\boldsymbol{\gamma}, \mathbf{b})$ are approximately dual frames for $L^{2}(\mathbb{R})$.

iii) Assume, additionally, for some $\delta$-separated set of time-sampling points $\left\{a_{k}\right.$ : $k \in \mathbb{Z}\}$ and constants $0<p_{U}, C_{L}, C_{U}<\infty$ such that for $\left.\left.p_{k} \in\right] 2, p_{U}\right] \subset \mathbb{R}$, $C_{k} \in\left[C_{L}, C_{U}\right]$ we have

$$
\left|g_{k}(t)\right| \leq C_{k}\left(1+\left|t-a_{k}\right|\right)^{-p_{k}} \text { for all } k \in \mathbb{Z}
$$

Then there exists a sequence $\left\{b_{k}^{0}\right\}_{k \in \mathbb{Z}}$, such that for all sequence $b_{k} \leq b_{k}^{0}, k \in \mathbb{Z}$, (17) holds.

Remark 1. If (17) holds, $\mathcal{G}(\boldsymbol{\gamma}, \mathbf{b})$ is called a single preconditioning dual system for $\mathcal{G}(\mathbf{g}, \mathbf{b})$. 
Proof. Since all frequency modulation parameters $b_{k}$ are taken from a closed interval in $\mathbb{R}^{+}$, the invertibility of $G_{0}^{g, g}$ is straightforward and the windows $\gamma_{k}$ are well defined. Moreover, since $\mathcal{G}(\mathbf{g}, \mathbf{b})$ is a Bessel sequence, so is $\mathcal{G}(\boldsymbol{\gamma}, \mathbf{b})$, with Bessel bound (ess inf $\left.G_{0}^{g, g}\right)^{-2} B$. Substituting $\gamma_{k}=\left(G_{0}^{g, g}\right)^{-1} g_{k}$ for $\gamma_{k}$ in the proof of Lemma 3.2, the first term in (13) vanishes and we obtain $(i)$ :

$$
\begin{aligned}
\left|\left\langle f-S_{g, \gamma} f, f\right\rangle\right| & \leq\left|\left\langle\left(G_{0}^{g, g}\right)^{-1} \sum_{l \in \mathbb{Z} \backslash\{0\}} \sum_{k \in \mathbb{Z}} b_{k}^{-1} g_{k}(\cdot) \overline{g_{k}\left(\cdot-l b_{k}^{-1}\right.}\right) f\left(\cdot-l b_{k}^{-1}\right), f\right\rangle \mid \\
& \leq\left(\operatorname{essinf} G_{0}^{g, g}\right)^{-1}\left\langle\sum_{l \in \mathbb{Z} \backslash\{0\}} \sum_{k \in \mathbb{Z}} b_{k}^{-1}\left|g_{k}(\cdot)\right|\left|g_{k}\left(\cdot-l b_{k}^{-1}\right)\right|\left|f\left(\cdot-l b_{k}^{-1}\right)\right|,|f|\right\rangle \\
& \leq\left(\operatorname{essinf} G_{0}^{g, g}\right)^{-1} R_{g, g}\|f\|_{2}^{2} .
\end{aligned}
$$

(ii) follows directly from Definition 3. Finally, (iii) follows from [8, Theorem 3.4], where it is shown that the assumptions (18) on the windows $g_{k}$ guarantee the existence of a sequence $b_{k}^{0}$ such that

$$
\left\langle S_{g, g} f, f\right\rangle\|f\|_{2}^{-2} \geq \operatorname{essinf} G_{0}^{g, g}-R_{g, g}>0
$$

Single preconditioning dual windows are a good choice for reconstruction, whenever the frame operator is close to diagonal. This is the case, if the original windows $g_{k}$ decay fast and frequency sampling is fast.

If the frame of interest is close to some other frame, which, ideally, is better understood, other approximate dual windows may be derived from this frame. The prototypical situation is a NSG frame which is close to a painless NSG frame in the sense of a small perturbation. Approximately dual frames in the context of perturbation theory were recently studied in [4]. In the following proposition we give error estimates for the reconstruction with approximately dual frames in such a situation. This provides different reconstruction methods apart from single preconditioning which was addressed in Proposition 4.1.

Proposition 4.2. Assume that $\mathcal{G}(\mathbf{g}, \mathbf{b})$ is a Bessel sequence with bound $B$ and that $\mathcal{G}(\mathbf{h}, \mathbf{b})$ is a NSG frame with lower and upper frame bound $A_{h}$ and $B_{h}$, respectively. We set $\psi_{k}=h_{k}-g_{k}$ and define the following windows:

$$
\begin{aligned}
& \text { (a) } \gamma_{k, l}^{1}=S_{h, h}^{-1} h_{k, l} \quad \text { (canonical dual of } h_{k, l} \text { ) } \\
& \text { (b) } \gamma_{k, l}^{2}=S_{h, h}^{-1} g_{k, l}
\end{aligned}
$$

Then the following hold: 
(i)

$$
\left\|I-S_{g, \gamma^{1}}\right\| \leq A_{h}^{-1 / 2}\left\|C_{\psi}\right\|
$$

If $\operatorname{ess} \sup \sum_{l \in \mathbb{Z}} G_{l}^{\psi, \psi}<A_{h}$, then $\mathcal{G}\left(\boldsymbol{\gamma}^{1}, \mathbf{b}\right)$ and $\mathcal{G}(\mathbf{g}, \mathbf{b})$ are approximately dual frames.

(ii)

$$
\left\|I-S_{g, \gamma^{2}}\right\| \leq A_{h}^{-1}\left(\sqrt{B_{h}}+\sqrt{B}\right)\left\|C_{\psi}\right\| .
$$

If $\operatorname{ess} \sup \sum_{l \in \mathbb{Z}} G_{l}^{\psi, \psi}<\frac{A_{h}^{2}}{\left(\sqrt{B_{h}}+\sqrt{B}\right)^{2}}$, then $\mathcal{G}\left(\gamma^{2}, \mathbf{b}\right)$ and $\mathcal{G}(\mathbf{g}, \mathbf{b})$ are approximately dual frames.

Remark 2. The first statement of Proposition 4.2 is contained in [4].

According to [8], the assumption that ess sup $\sum_{l \in \mathbb{Z}} G_{l}^{\psi, \psi}<A_{h}$ can be satisfied if the functions $\psi_{k}$ decay polynomially, i.e., $\left|\psi_{k}(t)\right| \leq C_{k}(1+|t|)^{-p_{k}}$ with appropriate constants $C_{k}$ and decay rates $p_{k}>1$.

Proof. From (17) it follows that $\left\|C_{\psi}\right\|^{2} \leq \operatorname{ess} \sup \sum_{l \in \mathbb{Z}} G_{l}^{\psi, \psi}$. The same estimate holds for $\left\|U_{\psi}\right\|^{2}$.

Since $\mathcal{G}(\mathbf{h}, \mathbf{b})$ is a frame with canonical dual frame $\mathcal{G}\left(\boldsymbol{\gamma}^{1}, \mathbf{b}\right)$, an upper frame bound of $\mathcal{G}\left(\boldsymbol{\gamma}^{1}, \mathbf{b}\right)$ is given by $A_{h}^{-1}$. We thus obtain (i) as follows:

$$
\left\|I-S_{g, \gamma^{1}}\right\|=\left\|U_{\gamma^{1}} C_{h}-U_{\gamma^{1}} C_{g}\right\| \leq\left\|U_{\gamma^{1}}\right\|\left\|C_{\psi}\right\| \leq A_{h}^{-1 / 2}\left\|C_{\psi}\right\|
$$

If ess $\sup \sum_{l \in \mathbb{Z}} G_{l}^{\psi, \psi}<A_{h}$, then $\left\|I-S_{g, \gamma^{1}}\right\|<1$ and $\mathcal{G}\left(\gamma^{1}, \mathbf{b}\right)$ and $\mathcal{G}(\mathbf{g}, \mathbf{b})$ are approximately dual frames as claimed.

To show $(i i)$, we note that $U_{\gamma^{2}}=S_{h, h}^{-1} U_{g}$ and thus

$$
\begin{aligned}
\left\|I-S_{g, \gamma^{2}}\right\| & =\left\|S_{h, h}^{-1} S_{h, h}-S_{h, h}^{-1} S_{g, g}\right\|=\left\|S_{h, h}^{-1}\left(U_{h} C_{h}-U_{g} C_{g}\right)\right\| \\
& \leq\left\|S_{h, h}^{-1}\right\|\left\|U_{h} C_{h}-U_{g} C_{h}+U_{g} C_{h}-U_{g} C_{g}\right\|=A_{h}^{-1}\left\|U_{\psi} C_{h}-U_{g} C_{\psi}\right\| \\
& \leq A_{h}^{-1}\left\|C_{\psi}\right\|\left(\sqrt{B_{h}}+\sqrt{B}\right)
\end{aligned}
$$

and (24) follows.

\subsection{Perturbation of painless nonstationary Gabor frames}

If a NSG system can be derived as a perturbation of a painless NSG frame, the approximately dual windows given in Proposition 4.2 are particularly simple to compute. In this situation, the frame $\mathcal{G}(\mathbf{h}, \mathbf{b})$ is the painless frame $\mathcal{G}\left(\mathbf{g}^{\mathbf{o}}, \mathbf{b}\right)$ and the frame operator 
$S_{h, h}=S_{g^{o}, g^{o}}$ is the multiplication operator $G_{0}^{g^{o}, g^{o}}$. Moreover, in this particular case, the approximately dual frames, given by $\gamma_{k, l}^{1}=\left(G_{0}^{g^{o}, g^{o}}\right)^{-1} g_{k, l}^{o}$ and $\gamma_{k, l}^{2}=\left(G_{0}^{g^{o}, g^{o}}\right)^{-1} g_{k, l}$ are NSG frames. This is a very important asset, since for NSG frames fast algorithms for analysis and reconstruction using FFT exist.

In 88 we constructed a special class of NSG frames, arising from painless NSG frames, which we introduce next.

Definition 4 (Almost painless NSG frames). Let $\mathcal{G}(\mathbf{g}, \mathbf{b})$ be a NSG system, assume that the windows $g_{k}$ are essentially bounded away from zero on the intervals $I_{k}=$ $\left[a_{k}-\left(2 b_{k}\right)^{-1}, a_{k}+\left(2 b_{k}\right)^{-1}\right]$ and set $g_{k}^{o}=g_{k} \chi_{I_{k}}$. If $\mathcal{G}\left(\mathbf{g}^{\mathbf{o}}, \mathbf{b}\right)$ is a (painless) frame for $L^{2}(\mathbb{R})$, then we call the system $\mathcal{G}(\mathbf{g}, \mathbf{b})$ an almost painless $N S G$ system (or frame).

For almost painless NSG systems, the estimates given in Proposition 4.2 can be written more explicitly.

Corollary 4.3. Assume that $\mathcal{G}(\mathbf{g}, \mathbf{b})$ is an almost painless $N S G$ system and let $A_{0}=$ $\operatorname{essinf} G_{0}^{g^{o}, g^{\circ}}$ to be the lower frame bound of the painless frame $\mathcal{G}\left(\mathbf{g}^{\mathbf{o}}, \mathbf{b}\right)$ and $g_{k}^{r}=$ $g_{k}-g_{k} \chi_{I_{k}}$. Then the following hold:

(i) for $\gamma_{k}^{1}=\left(G_{0}^{g^{o}, g^{o}}\right)^{-1} g_{k}^{o}$,

$$
\left\|I-S_{g, \gamma^{1}}\right\| \leq A_{0}^{-1} \sqrt{R_{g^{r}, g^{o}} \cdot R_{g^{o}, g^{r}}}
$$

(ii) for $\gamma_{k}^{2}=\left(G_{0}^{g^{o}, g^{o}}\right)^{-1} g_{k}$

$$
\left\|I-S_{g, \gamma^{2}}\right\| \leq A_{0}^{-1}\left(R_{g^{r}, g^{o}}+R_{g^{o}, g^{r}}+\operatorname{ess} \sup \sum_{l \in \mathbb{Z}} G_{l}^{g^{r}, g^{r}}\right)
$$

Proof. The estimates follow from Lemma 3.2. First, substituting $\gamma_{k}^{1}=\left(G_{0}^{g^{o}, g^{o}}\right)^{-1} g_{k}^{o}$ for $\gamma_{k}$ in (12), the first term vanishes, since $\sum_{k \in \mathbb{Z}} b_{k}^{-1} \overline{g_{k}} g_{k}^{o}=G_{0}^{g^{o}, g^{o}}$, and we obtain

$$
\left\|I-S_{g, \gamma^{1}}\right\| \leq \sqrt{R_{g, \gamma^{1}} \cdot R_{\gamma^{1}, g}} \leq\left(\operatorname{essinf} G_{0}^{g^{o}, g^{o}}\right)^{-1} \sqrt{R_{g^{r}, g^{o}} \cdot R_{g^{o}, g^{r}}},
$$

since

$$
\begin{aligned}
R_{g, \gamma^{1}} & =\operatorname{ess} \sup \sum_{l \in \mathbb{Z} \backslash\{0\}} \sum_{k \in \mathbb{Z}} b_{k}^{-1}\left|g_{k}\left(\cdot-l b_{k}^{-1}\right)\right|\left|\left(G_{0}^{g^{o}, g^{o}}\right)^{-1} g_{k}^{o}(\cdot)\right| \\
& \leq\left(\operatorname{essinf} G_{0}^{g^{o}, g^{o}}\right)^{-1} \cdot \operatorname{ess} \sup \sum_{l \in \mathbb{Z} \backslash\{0\}} \sum_{k \in \mathbb{Z}} b_{k}^{-1}\left|g_{k}^{r}\left(\cdot-l b_{k}^{-1}\right)\right|\left|g_{k}^{o}(\cdot)\right| \\
& =\left(\operatorname{essinf} G_{0}^{g^{o}, g^{o}}\right)^{-1} R_{g^{r}, g^{o}},
\end{aligned}
$$


similarly for $R_{\gamma^{1}, g}$.

For (ii) we substitute $\gamma_{k}^{2}=\left(G_{0}^{g^{o}, g^{\circ}}\right)^{-1} g_{k}$ for $\gamma$ in the proof of Lemma 3.2, $g_{k}^{o}+g_{k}^{r}$ for $g_{k}$ and use the fact that $g_{k}^{o}$ and $g_{k}^{r}$ have disjoint supports. Then, since $\overline{g_{k}^{o}\left(\cdot-l b_{k}^{-1}\right)} g_{k}^{o}(\cdot)$ is zero for $l \neq 0$, using (14) we obtain that

$$
\begin{aligned}
\left|\left\langle f-S_{g, \gamma^{2}} f, f\right\rangle\right| & =\left|\left\langle f-\sum_{k \in \mathbb{Z}} \sum_{l \in \mathbb{Z}} \overline{g_{k}\left(\cdot-l b_{k}^{-1}\right)}\left(G_{0}^{g^{o}, g^{o}}\right)^{-1} g_{k}(\cdot) f\left(\cdot-l b_{k}^{-1}\right), f\right\rangle\right| \\
& \leq\left|\left\langle f-\left(G_{0}^{g^{o}, g^{o}}\right)^{-1} \sum_{k \in \mathbb{Z}} b_{k}^{-1}\left|g_{k}^{o}\right|^{2} f, f\right\rangle\right| \\
& +\left(\operatorname{essinf}\left(G_{0}^{g^{o}, g^{o}}\right)^{-1}\left(R_{g^{r}, g^{o}}+R_{g^{o}, g^{r}}+\left\|S_{g^{r}, g^{r}}\right\|\right)\|f\|_{2}^{2} .\right.
\end{aligned}
$$

The first term vanishes and by (17), $\left\|S_{g^{r}, g^{r}}\right\| \leq \operatorname{ess} \sup \sum_{l \in \mathbb{Z}} G_{l}^{g^{r}, g^{r}}$.

\section{$5 \quad$ Examples}

We present two examples to illustrate our theory. In the first example we deal with almost painless frames using Gaussian windows. We consider three different approximately dual frames and check their performance in terms of reconstruction.

In both examples, we consider a basic window and dilations by 2 and $\frac{1}{2}$, respectively. Since the dilation parameters take only three different values, there are three kinds of windows, with support size $1 / 2,1$ and 2 , respectively. Note that, while theoretically possible, sudden changes in the shape and width of adjacent windows turn out to be undesirable for applications, hence we only allow for stepwise change in dilation parameters.

Example 5.1. Let $s_{k} \in\{-1,0,1\}$ with $\left|s_{k}-s_{k-1}\right| \in\{0,1\}$ for all $k \in \mathbb{Z}$. We consider a sequence of windows $g_{k}$ that are translated and dilated versions of the Gaussian window $g(t)=e^{-\pi[\sigma t]^{2}}: g_{k}(t)=T_{a_{k}} \sqrt{b_{k}} g\left(b_{k} t\right)=\sqrt{b_{k}} g\left(b_{k}\left(t-a_{k}\right)\right)$, with $\sigma=2.5$, $b_{k}=2^{s_{k}}, a_{0}=0$ and for all $k \in \mathbb{Z}$

$$
\begin{aligned}
& a_{k+1}=a_{k}+\left(2 b_{k}\right)^{-1} \quad \text { if } \quad s_{k}=s_{k+1}, \\
& a_{k+1}=a_{k}+\left(3 b_{k+1}\right)^{-1} \quad \text { if } \quad s_{k}>s_{k+1} \text {, } \\
& a_{k+1}=a_{k}+\left(3 b_{k}\right)^{-1} \quad \text { if } \quad s_{k}<s_{k+1} \text {. }
\end{aligned}
$$

Here, $b_{L}=1 / 2, b_{U}=2$ and the $\left\{a_{k}: k \in \mathbb{Z}\right\}$ are separated with minimum distance $\delta=1 / 4$. We arrange the windows as follows: after each change of window size, no change is allowed in the next step; in other words, each window has at least one neighbor of the same size. 
Let $I_{k}=\left[a_{k}-\left(2 b_{k}\right)^{-1}, a_{k}+\left(2 b_{k}\right)^{-1}\right]$ and define a new set of windows by $g_{k}^{o}(t)=$ $g_{k}(t) \chi_{I_{k}}$. Then $\left\{M_{l b_{k}} g_{k}^{o}: k, l \in \mathbb{Z}\right\}$ is a painless nonstationary Gabor frame with lower frame bound $A_{0}=0.1718$.

The system $\mathcal{G}(\mathbf{g}, \mathbf{b})$ arises from the painless frame $\mathcal{G}\left(\mathbf{g}^{\mathbf{o}}, \mathbf{b}\right)$, and therefore we are interested in the approximate dual windows proposed in Corollary 4.3. We first consider $\gamma_{k}^{1}=\left(G_{0}^{g^{o}, g^{o}}\right)^{-1} g_{k}^{o}$, the canonical dual frame of $\mathcal{G}\left(\mathbf{g}^{\mathbf{o}}, \mathbf{b}\right)$. According to (29) , we need to calculate $R_{g^{r}, g^{o}}$ and $R_{g^{o}, g^{r}}$ in order to obtain an estimate of the reconstruction error.

Claim 1: $R_{g^{r}, g^{\circ}} \leq 0.00827+\mathcal{O}\left(10^{-6}\right)$.

For fixed $k \in \mathbb{Z}$,

$$
b_{k}^{-1}\left|g_{k}^{o}(t)\right| \sum_{l \in \mathbb{Z} \backslash\{0\}}\left|g_{k}^{r}\left(t-l b_{k}^{-1}\right)\right|=b_{k}^{-1 / 2}\left|g_{k}^{o}(t)\right| \sum_{l \in \mathbb{Z}} b_{k}^{-1 / 2}\left|g_{k}^{r}\left(t-l b_{k}^{-1}\right)\right|
$$

since $g_{k}^{r}$ and $g_{k}^{o}$ have disjoint support. Then, due to $b_{k}^{-1}$-periodicity of $\sum_{l \in \mathbb{Z}} b_{k}^{-1 / 2} \mid g_{k}^{r}(t-$ $\left.l b_{k}^{-1}\right) \mid$, we have

$$
\begin{aligned}
R_{g^{r}, g^{o}} & =\operatorname{ess} \sup \sum_{k \in \mathbb{Z}} b_{k}^{-1 / 2}\left|g_{k}^{o}(\cdot)\right| \sum_{l \in \mathbb{Z}} b_{k}^{-1 / 2}\left|g_{k}^{r}\left(\cdot-l b_{k}^{-1}\right)\right| \\
& \leq \operatorname{ess} \sup \sum_{k \in \mathbb{Z}} b_{k}^{-1 / 2}\left|g_{k}^{o}(\cdot)\right| \cdot \operatorname{ess}^{\sup _{t \in \mathcal{I}_{k}}} \sum_{l \in \mathbb{Z}} b_{k}^{-1 / 2}\left|g_{k}^{r}\left(t-l b_{k}^{-1}\right)\right|
\end{aligned}
$$

In order to obtain more accurate estimates, we split $I_{k}$ by setting $I_{k}^{+}=\left[a_{k}, a_{k}+\right.$ $\left.\left(2 b_{k}\right)^{-1}\right]$ and $I_{k}^{-}=\left[a_{k}-\left(2 b_{k}\right)^{-1}, a_{k}\right]$ and estimate expression (32) by its values at the end points of $I_{k}^{+}$or $I_{k}^{-}$, respectively. We observe that, for $t \in I_{k}^{+}$:

$$
\begin{aligned}
\sum_{l \in \mathbb{Z}} b_{k}^{-1 / 2}\left|g_{k}^{r}\left(t-l b_{k}^{-1}\right)\right| & =\sum_{l=1}^{\infty}\left[e^{-\pi\left[\sigma b_{k}\left(t-a_{k}-l b_{k}^{-1}\right)\right]^{2}}+e^{-\pi\left[\sigma b_{k}\left(t-a_{k}+l b_{k}^{-1}\right)\right]^{2}}\right] \\
& \leq \sum_{l=1}^{\infty} e^{-\pi[\sigma l]^{2}}+\sum_{l=1}^{\infty} e^{-\pi[\sigma(2 l-1) / 2]^{2}} \leq 0.00738+\mathcal{O}\left(10^{-6}\right) .
\end{aligned}
$$

By the symmetry of $g_{k}^{r}$ with respect to $a_{k}$, an analogous estimate holds for $t \in I_{k}^{-}$. Furthermore, due to the arrangement of the windows $g_{k}$, ess $\sup \sum_{k \in \mathbb{Z}} b_{k}^{-1 / 2}\left|g_{k}^{o}(\cdot)\right| \leq$ 1.1206 and Claim 1 follows.

Claim 2: $R_{g^{o}, g^{r}} \leq 0.0157+\mathcal{O}\left(10^{-6}\right)$.

Observe that $\sum_{l \in \mathbb{Z} \backslash\{0\}} b_{k}^{-1 / 2}\left|g_{k}^{o}\left(t-l b_{k}^{-1}\right)\right| \leq\left\|b_{k}^{-1 / 2} g_{k}^{o}\right\|_{\infty}=1$. Then, with $I_{m}^{+}$and 


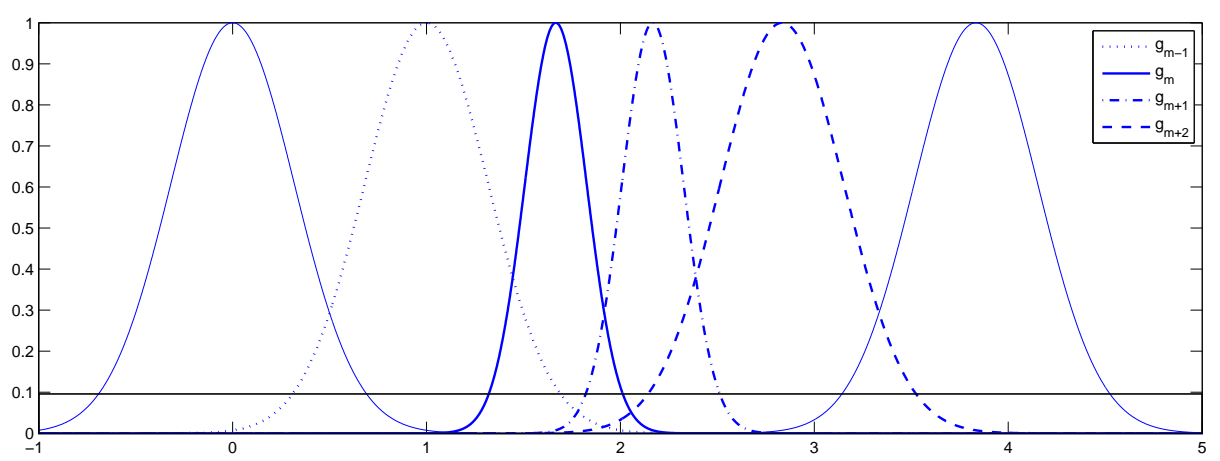

Figure 1: An example of an arrangement of windows $g_{k}$.

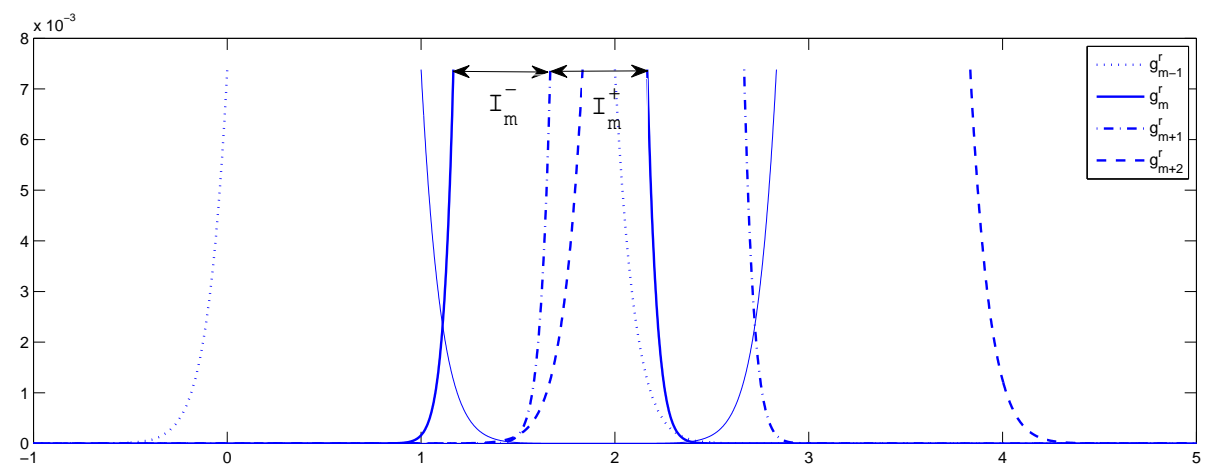

Figure 2: A zoom-in on the tails $g_{k}^{r}$ in the arrangement of Fig. 1.

$I_{m}^{-}$defined as before:

$$
\begin{aligned}
& R_{g^{o}, g^{r}}=\operatorname{ess} \sup \sum_{k \in \mathbb{Z}} b_{k}^{-1 / 2}\left|g_{k}^{r}(\cdot)\right| \sum_{l \in \mathbb{Z} \backslash\{0\}} b_{k}^{-1 / 2}\left|g_{k}^{o}\left(\cdot-l b_{k}^{-1}\right)\right| \leq \operatorname{ess} \sup \sum_{k \in \mathbb{Z}} b_{k}^{-1 / 2}\left|g_{k}^{r}(\cdot)\right| \\
& \leq \sup _{m \in \mathbb{Z}}\left\{\operatorname{ess}_{\sup } \sum_{t \in I_{m}^{+}} \sum_{k \in \mathbb{Z}} b_{k}^{-1 / 2}\left|g_{k}^{r}(t)\right|, \operatorname{ess} \sup _{t \in I_{m}^{-}} \sum_{k \in \mathbb{Z}} b_{k}^{-1 / 2}\left|g_{k}^{r}(t)\right|\right\}
\end{aligned}
$$

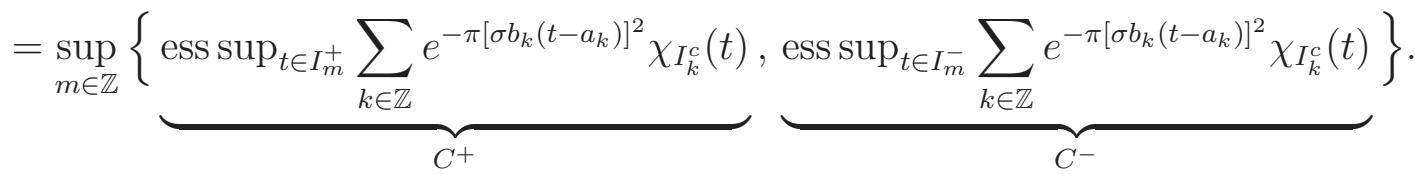

We bound $C^{+}$and $C^{-}$by their maximal values on $I_{m}^{+}$, respectively $I_{m}^{-}$. The set $\left\{a_{k}: k \in \mathbb{Z}\right\}$ is $\delta$-separated, hence $\left|a_{k}-a_{m}\right| \geq|k-m| \delta$. By the arrangement of the windows, there are at most two windows $g_{k}^{r}$ which assume their maximum $e^{-\pi[\sigma / 2]^{2}}$ in the interval $I_{m}^{+}$or in $I_{m}^{-}$. Without loss of generality we assume that the two maximal values occur in $I_{m}^{+}$, corresponding to the windows $g_{m-1}^{r}$ and $g_{m+2}^{r}$. Then, $g_{m+1}^{r}, g_{m}^{r}$ 
are zero in $I_{m}^{+}$, cf. Figure 2 for an example situation. Therefore,

$$
\begin{aligned}
C^{+} & \leq \sum_{k>m+2} e^{-\pi\left[\sigma b_{k}\left(a_{m}+\left(2 b_{m}\right)^{-1}-a_{k}\right)\right]^{2}}+2 e^{-\pi\left[\sigma b_{k}\left(2 b_{k}\right)^{-1}\right]^{2}}+\sum_{k<m-1} e^{-\pi\left[\sigma b_{k}\left(a_{m}-a_{k}\right)\right]^{2}} \\
& \leq 2\left(e^{-\pi[\sigma / 2]^{2}}+\sum_{k>0 ; k b_{k}>2} e^{-\pi\left[\sigma b_{k} k \delta\right]^{2}}\right)
\end{aligned}
$$

Since we assumed that two windows $g_{k}^{r}$ reached their maximum in $I_{m}^{+}, C^{-} \leq C^{+}$. As before, the bound from Claim 2 follows by numerical calculations.

In summary, due to (29), Claim 1 and 2 and the lower frame bound $A_{0}=0.1718$, the reconstruction error using the approximate duals $\gamma_{k}^{1}$ is bounded by

$$
\left\|I-S_{g, \gamma^{1}}\right\| \leq 0.0663+\mathcal{O}\left(10^{-6}\right)
$$

Another choice of approximate dual system are the windows $\gamma_{k}^{2}=\left(G_{0}^{g^{o}, g^{o}}\right)^{-1} g_{k}$. In this setting we use (30) to derive

$$
\left\|I-S_{g, \gamma^{2}}\right\| \leq A_{0}^{-1}\left(R_{g^{r}, g^{o}}+R_{g^{o}, g^{r}}+\operatorname{ess} \sup \sum_{l \in \mathbb{Z}} G_{l}^{g^{r}, g^{r}}\right) \leq 0.1402+\mathcal{O}\left(10^{-6}\right)
$$

since, by previous calculations,

$$
\begin{aligned}
\operatorname{ess} \sup \sum_{l \in \mathbb{Z}} G_{l}^{g^{r}, g^{r}} & =\operatorname{ess} \sup \sum_{k \in \mathbb{Z}} b_{k}^{-1 / 2}\left|g_{k}^{r}(t)\right| \sum_{l \in \mathbb{Z}} b_{k}^{-1 / 2}\left|g_{k}^{r}\left(t-l b_{k}^{-1}\right)\right| \\
& \leq \operatorname{ess} \sup \sum_{k \in \mathbb{Z}} b_{k}^{-1 / 2}\left|g_{k}^{r}(t)\right| \cdot \operatorname{ess} \sup \sum_{l \in \mathbb{Z}} b_{k}^{-1 / 2}\left|g_{k}^{r}\left(t-l b_{k}^{-1}\right)\right| \\
& \leq 0.0001158+\mathcal{O}\left(10^{-6}\right) .
\end{aligned}
$$

As a third choice of approximate dual windows we consider single preconditioning windows $\gamma_{k}=\left(G_{0}^{g, g}\right)^{-1} g_{k}$ introduced in Proposition 4.1. It can easily be seen that

$$
R_{g, g} \leq R_{g^{o}, g^{r}}+R_{g^{r}, g^{o}}+R_{g^{r}, g^{r}} \leq R_{g^{o}, g^{r}}+R_{g^{r}, g^{o}}+\operatorname{ess} \sup \sum_{l \in \mathbb{Z}} G_{l}^{g^{r}, g^{r}}
$$

and, by previous calculations, it follows

$$
R_{g, g} \leq 0.0241+\mathcal{O}\left(10^{-6}\right)<A_{0} \leq \operatorname{essinf} G_{0}^{g, g}
$$

Therefore, by Proposition 4.1 (ii), $\mathcal{G}(\mathbf{g}, \mathbf{b})$ and $\mathcal{G}(\gamma, \mathbf{b})$ are approximately dual frames. Moreover

$$
\left\|I-S_{g, \gamma}\right\| \leq \frac{R_{g, g}}{\operatorname{essinf} G_{0}^{g, g}} \leq \frac{R_{g, g}}{A_{0}} \leq 0.1402+\mathcal{O}\left(10^{-6}\right)
$$


Remark 3. Observe that from each of the approximately dual frame estimates given in the above example, the frame property of $\mathcal{G}(\mathbf{g}, \mathbf{b})$ follows.

Note that, from (34), the frame property of $\mathcal{G}(\mathbf{g}, \mathbf{b})$ may also be derived by applying results from perturbation theory cf. [3]. Indeed, since $\sum_{k, l \in \mathbb{Z}}\left|\left\langle f, g_{k, l}-g_{k, l}^{o}\right\rangle\right|^{2}=$ $\sum_{k, l \in \mathbb{Z}}\left|\left\langle f, g_{k, l}^{r}\right\rangle\right|^{2} \leq \operatorname{ess} \sup \sum_{l \in \mathbb{Z}} G_{l}^{g^{r}, g^{r}}\|f\|_{2}^{2}<A_{0}\|f\|_{2}^{2}$, it follows that $\mathcal{G}(\mathbf{g}, \mathbf{b})$ is a frame with a lower frame bound $A=0.1630$.

Example 5.2. In our second example, we turn to the situation mentioned in the introduction, namely, the construction of non-uniform filter banks with compactly supported, that is, FIR filters, via NSG frames. In this situation, the frame operator $S$ does not have a Walnut-like structure as given in (5) on the time side. However, $S$ may be considered on the frequency side by applying a Fourier transform. Then, we encounter the same structure as before and may exploit the developed techniques to deduce the frame property and to construct approximate dual frames for reconstruction. The situation is schematically depicted in Figure 3 ,
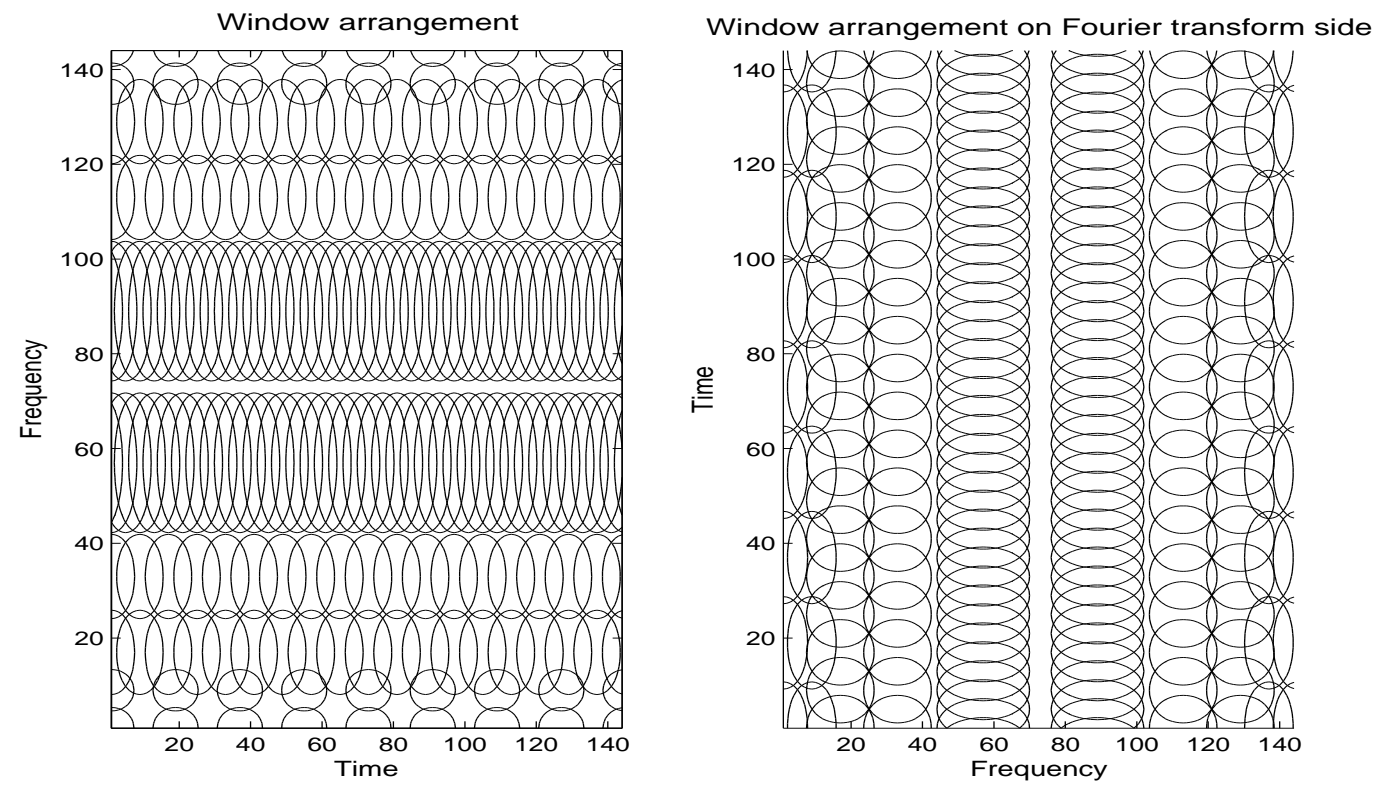

Figure 3: An example for the arrangement of dilated windows in Example 2

It is obvious that, while, in the time domain, various windows $g_{k}$ with different pass-bands, are shifted to obtain the overall system, applying a Fourier transform yields the known situation: various windows $\hat{g}_{k}$ are modulated to create an NSG system.

On the other hand, we are interested in using FIR filters, that is, the windows $g_{k}$ 
are all compactly supported, hence not bandlimited. Similar to the construction in Example 5.1, we can cut the windows $\hat{g}_{k}$ to obtain a painless NSG reference frame. More precisely, we consider the family of windows $g_{k}$ and a vector of corresponding time-shift parameters $a_{k}$. Then, we set $g_{k, l}=T_{a_{k} l} g_{k}$ and are interested in the frame property of the set of functions $\left\{g_{k, l}: k, l \in \mathbb{Z}\right\}$. Considering, due to the lack of structure on the time side as mentioned above, the corresponding frame operator on the frequency side corresponds to investigating the operator $\mathcal{F S F}^{*}$, which acts on the Fourier transform of a signal of interest. In other words, we are now dealing with the set of functions $\left\{\mathcal{F}\left(g_{k, l}\right)=M_{a_{k} l} \hat{g}_{k}: k, l \in \mathbb{Z}\right\}$.

For the current example, we consider Hanning windows $h_{k}$ and, as in the previous example, apply dilations by $2^{-1}$ and 2 , respectively, to obtain various time- and frequency resolutions. The time-shift parameters $a_{k}$ are chosen in parallel to the choice of the frequency-shift parameters $b_{k}$ in Example 5.1 .

Given the explicit knowledge of the spectral properties of the Hanning windows, explicit error estimates can be derived in a similar manner as in the previous example. Here, we also numerically calculate the errors resulting from reconstruction by means of the three different proposed approximate dual systems. We use the same nomenclature as before, that is, $\gamma_{k}^{1}, \gamma_{k}^{2}$ and $\gamma_{k}^{3}$ denote the canonical duals of the painless frame, the set of windows $\left(G_{0}^{g^{o}, g^{o}}\right)^{-1} g_{k}$ and the single preconditioning windows, respectively. Then

1. $\left\|I-S_{g, \gamma^{1}}\right\|=0.0210$

2. $\left\|I-S_{g, \gamma^{2}}\right\|=0.0407$

3. $\left\|I-S_{g, \gamma^{3}}\right\|=0.0407$

As before, the canonical duals of the painless frame provide the best approximate reconstruction.

The set of windows $\hat{g}_{k}$ used in this example, together with their true, single preconditioning duals $\gamma_{k}^{3}$ and the canonical duals of the corresponding painless frame, $\gamma_{k}^{1}$, are depicted in Figure 4. On the right plots, zoom-ins are shown to better compare the detailed behavior.

A comparison between a basic window from the true dual frame and the preconditioning duals $\gamma_{k}^{3}$ and the true dual and the approximate dual $\gamma_{k}^{1}$ is depicted in Figures 5 and 6, respectively, in both the frequency domain (upper plot) and the time domain (lower plot). It should be noted that, while compactly supported in frequency, $\gamma_{k}^{1}$ 
still have better decay in the time domain than both the true dual windows and the other approximate dual windows.

\section{References}

[1] Peter Balazs, Monika Dörfler, Florent Jaillet, Nicki Holighaus, and Gino Angelo Velasco. Theory, implementation and applications of nonstationary Gabor Frames. J. Comput. Appl. Math., 236:'1481-1496, 2011.

[2] Peter Balazs, Hans G. Feichtinger, M. Hampejs, and Guenther Kracher. Double preconditioning for Gabor frames. IEEE Trans. Signal Process., 54(12):45974610, December 2006.

[3] Ole Christensen. Perturbation of frames and applications to Gabor frames. In Hans G. Feichtinger and T. Strohmer, editors, Gabor Analysis and Algorithms: Theory and Applications, pages 193-209. Birkhäuser Boston, 1998.

[4] Ole Christensen and R. Laugesen. Approximately dual frame pairs in Hilbert spaces and applications to Gabor frames. Sampl. Theory Signal Image Process., 2010.

[5] Charles K. Chui and X. Shi. Bessel sequences and affine frames. Appl. Comput. Harmon. Anal., 01.Jän:29-49, 1994.

[6] Ingrid Daubechies, A. Grossmann, and Y. Meyer. Painless nonorthogonal expansions. J. Math. Phys., 27(5):1271-1283, May 1986.

[7] Monika Dörfler. Quilted Gabor frames - A new concept for adaptive timefrequency representation. Advances in Applied Mathematics, 47(4):668 - 687, Oct. 2011.

[8] Monika Dörfler and Ewa Matusiak. Nonstationary Gabor Frames - Existence and Construction. preprint, http://arxiv.org/abs/1112.5262, 2012.

[9] Karlheinz Gröchenig. Foundations of Time-Frequency Analysis. Appl. Numer. Harmon. Anal. Birkhäuser Boston, 2001.

[10] Nicki Holighaus, Monika Dörfler, Gino Velasco, and Thomas Grill. A framework for invertible, real-time constant-Q transforms. IEEE 
Transactions on Audio, Speech and Language Processing, to appear, http://www.univie.ac.at/nonstatgab/slicq/, 2012.

[11] Florent Jaillet. Représentation et traitement temps-fréquence des signaux audionumériques pour des applications de design sonore. $\mathrm{PhD}$ thesis, Université de la Méditerranée - Aix-Marseille II, 2005.

[12] Florent Jaillet and Bruno Torrésani. Timefrequency jigsaw puzzle: adaptive multiwindow and multilayered Gabor expansions. Int. J. Wavelets Multiresolut. Inf. Process., 2:293-316, 2007.

[13] A.J.E.M. Janssen and Peter L. Sondergaard. Iterative algorithms to approximate canonical Gabor windows: Computational aspects. J. Fourier Anal. Appl., 13(2):211-241, 2007.

[14] Marco Liuni, Peter Balazs, and Axel Röbel. Sound Analysis and Synthesis Adaptive in Time and Two Frequency Bands. In Proc. of the 14th Int. Conference on Digital Audio Effects (DAFx-11), Paris, France, September 19-23, volume accepted. ARI;NHG-coop, September 2011.

[15] José Luis Romero. Surgery of spline-type and molecular frames. J. Fourier Anal. Appl., 17:135 - 174, 2011.

[16] Peter Sondergaard. Efficient algorithms for the discrete Gabor transform with a long Fir window. J. Fourier Anal. Appl., 18(3):456-470, 2012.

[17] Thomas Strohmer. Approximation of dual Gabor frames, window decay, and wireless communications. Appl. Comput. Harmon. Anal., 11(2):243-262, 2001.

[18] Gino Angelo Velasco, Nicki Holighaus, Monika Dörfler, and Thomas Grill. Constructing an invertible constant-Q transform with non-stationary Gabor frames. Proceedings of DAFX11, 2011.

[19] Robert M. Young. An Introduction to Nonharmonic Fourier Series. Academic Press, Revised 1st edition edition, 2001. 
Original (almost painless) frame

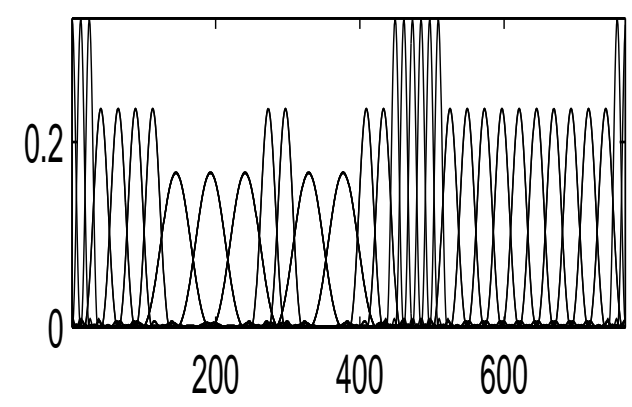

True dual frame

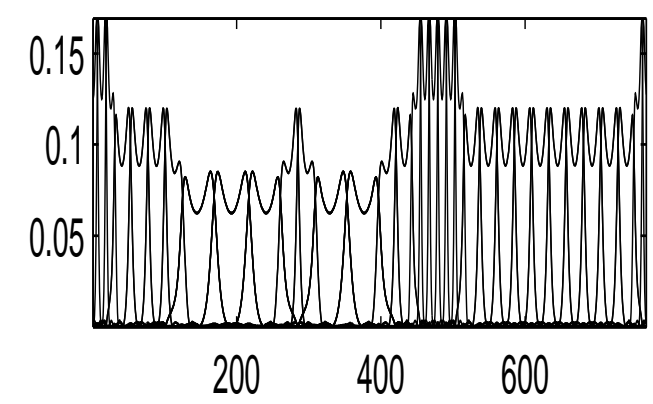

Single preconditioning dual frame $\left(\gamma^{3}\right)$

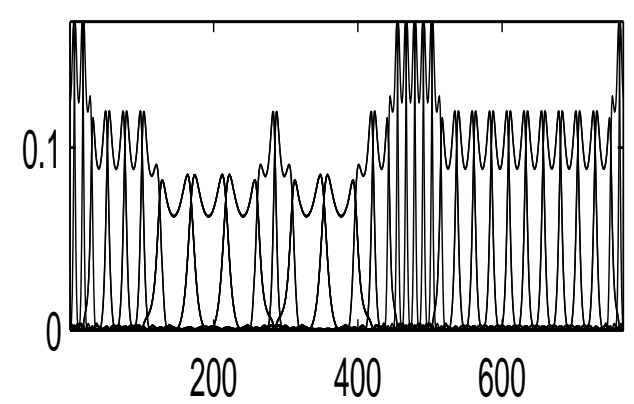

Canonical duals of painless frame $\left(\gamma^{\dagger}\right)$

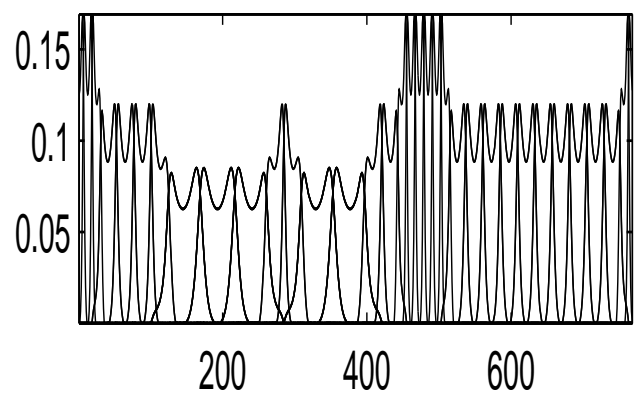

Original (almost painless) frame

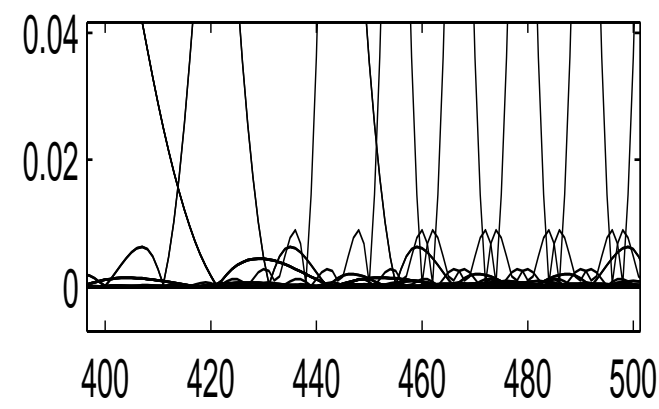

True dual frame

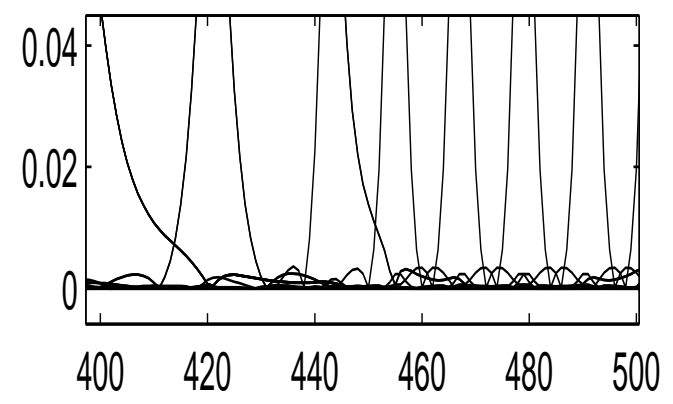

Single preconditioning dual frame $\left(\gamma^{3}\right)$

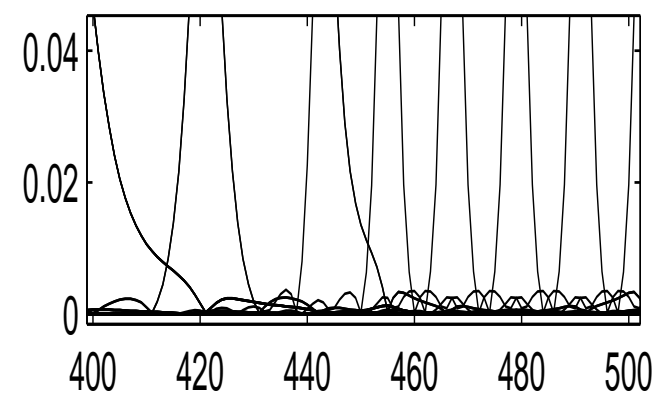

Canonical duals of painless frame $\left(\gamma^{1}\right)$

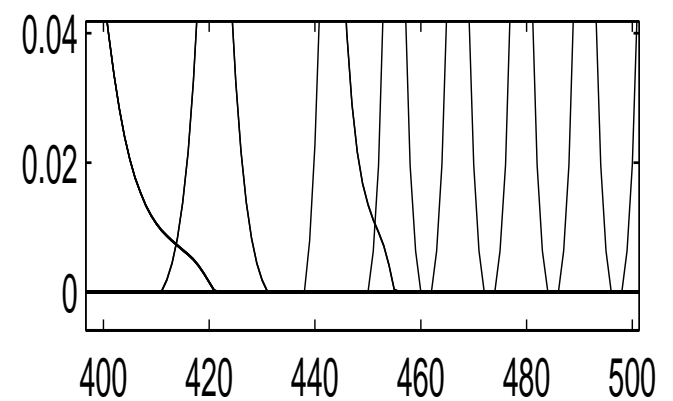

Figure 4: The original windows and the windows from various (approximate) dual frames. 

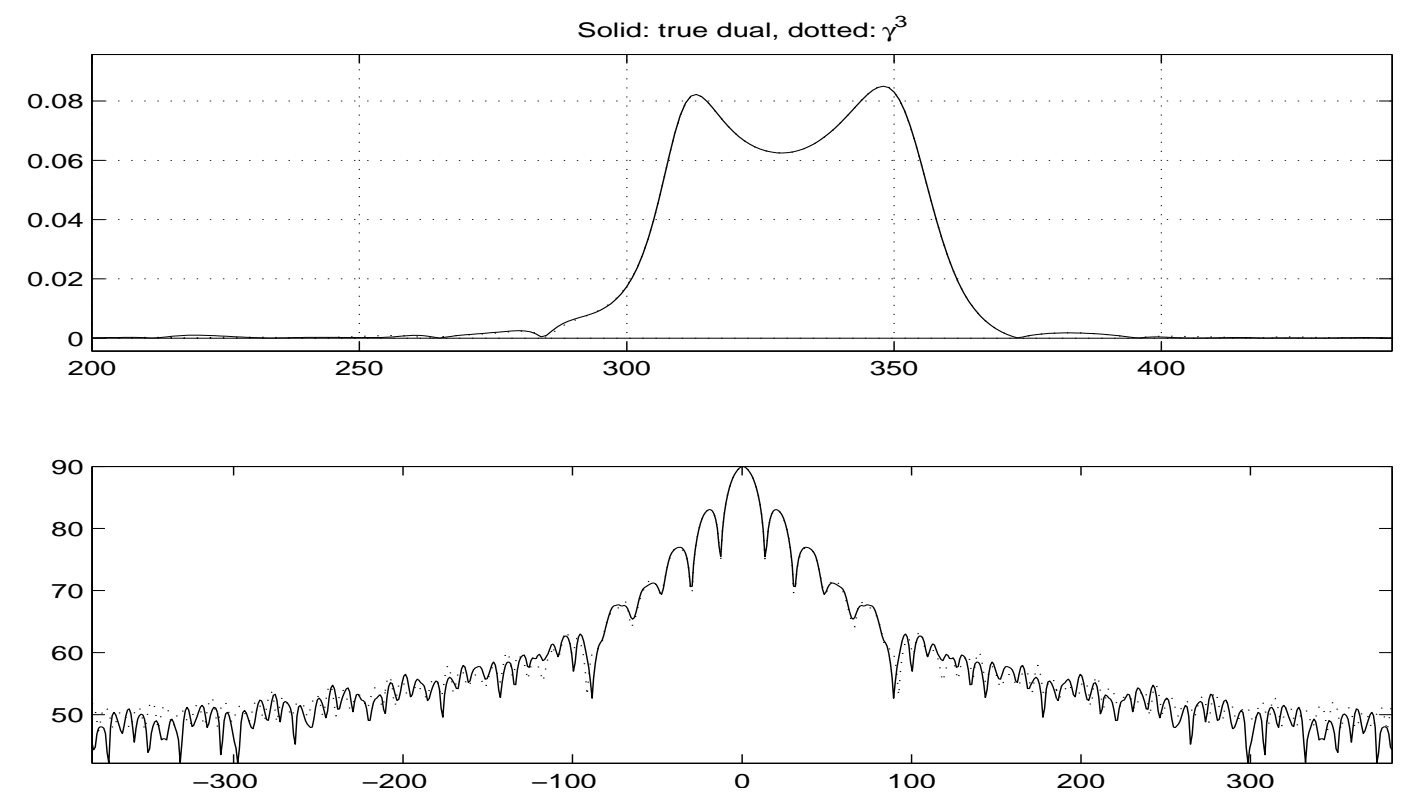

Figure 5: Comparison between true dual and single preconditioning dual $\gamma^{3}$.
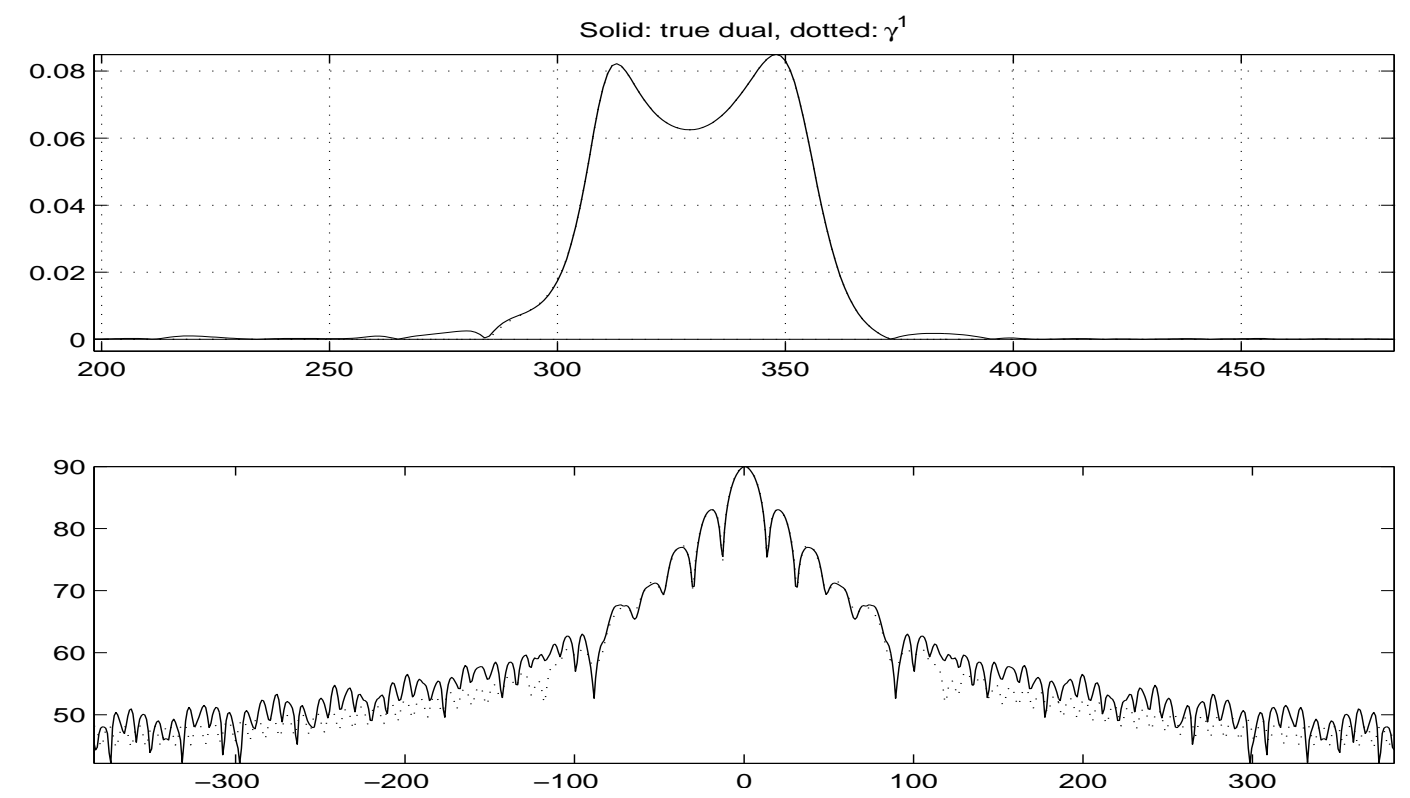

Figure 6: Comparison between true dual and painless approximate dual $\gamma^{1}$. 